\title{
Risk Factor for Periodontitis
}

\section{Saraswati Sachan}

\begin{abstract}
Periodontal disease possesses a significant challenge to the patient and the oral health care professional equally. Risk factor that are associated with periodontal disease must be properly identified and examine by professional before the patient is treated by periodontal treatment. It is important as how we educate a patient on risk management. This article offers strategies for professional to use and helps in establishing a balance preventive oral management plan. Pathogenesis of periodontal disease is more complex than the presence of virulent microorganism. Susceptibility to periodontitis varies between individuals who carry the same pathogenic micro flora.
\end{abstract}

\section{Introduction-}

All individuals are uniformly susceptible to developing periodontal disease. Accumulation of plaque, poor oral hygiene and occlusion trauma are sufficient to initiate periodontitis however during the past four decades it is believed that periodontal disease is caused by specific bacterial infections and that individuals are uniformly susceptible neither to these infections nor to the damage caused by them. So the main concentration is to find about the developing markers that allows identification of susceptible individuals prior to developing periodontitis and identifying risk factor that can be modified in order to prevent or alter the periodontal disease.

\section{Risk factors-}

Risk can be identified in terms of risk factor, determinant, indicators, and predictors.

A risk factor is any attribute, characteristic or exposure of individuals that increases the likelihood of developing a disease.it can be environmental, behavioural,or biologic factors.

Risk determinant should be reserved for that risk factor that cannot be modified.

Risk indicator have been identified in cross sectional studies but not confirmed through longitudinal studies.

Risk predictors, although associated with increased risk for disease, do not cause the disease.

\section{Risk factor-}

\begin{tabular}{|l|l|l|l|}
\hline Risk factor & Risk determinants & Risk indicator & Risk predictor \\
\hline Smoking & Genetic factor & HIV/AIDS & Bleeding on probing \\
\hline diabetes & Age & Osteoporosis & $\begin{array}{l}\text { Previous history of periodontal } \\
\text { disease }\end{array}$ \\
\hline Pathogenic bacteria & Gender & & \\
\cline { 1 - 2 } Microbial tooth deposit & Stress & & \\
\cline { 2 - 3 } & Socioeconomic status & \\
\hline
\end{tabular}

\section{Periodontitis -}

Periodontitis is a inflammatory diseases affecting the periodontium i.e.the tissue that surround and support the teeth. Periodontitis involves progressive loss of the alveolar bone around the teeth and if left untreated, can lead to the loosening and subsequent loss of teeth.

\section{Risk factor for periodontitis -}

Risk factors are biological related to occurrence of disease, but it may not be necessarily imply cause and effect.Risk factor may be broadly classified-

Systemic risk factor-factor that affect the host response to the plaque biofilm, upsetting the host microbial balance.

Local risk factor-factors that is local to the oral cavity and which may influence plaque accumulation or occlusal forces. $^{(1)}$

\section{Smoking-}

\section{Risk factor-}

The relationship between smoking and periodontal health was investigated in middle of the last century .Recently a wealth of epidemiology, clinical and in vitro studies have emerged that have provides evidence that smoking negatively impacts periodontal health and propose mechanism by which this may occurs 
(2) .Smoking inhibits immunological function and negativity affects immunoglobulin level which may increases susceptibility to typical and unusual microbial pathogens ${ }^{(3)}$.Chronic inhalation of cigarette smoke alters a wide range of immunological function, including innate and adaptive immune response. Cross sectional and longitudinal data shows that the risk of developing periodontal disease is measured by clinical attachment loss and alveolar bone loss increases with increased smoking. A no.of studies have established that smoking is associated with reduced gingival bleeding ${ }^{(4)}$.Studies have shown that smoking does not reduce the amount of plaque present and in fact smokers may experience less gingival bleeding than non-smokers with lower plaque indexes ${ }^{(5)}$.Reduced bleeding reflect an underlying disruption of the immune response and this may account for the increased loss of clinical attachment and alveolar bone. These finding suggest the use of gingival bleeding as an indicator of gingival inflammation when assessing a smoker's periodontal status.Periodontal treatment tends to be less likely to be successful in smokers than in non-smokers ${ }^{(6)}$.In vitro studies have shown altered gingival cervical fluid inflammatory cytokine profiles ,immune cellfunction ${ }^{(7)}$.and altered proteolytic regulation in smokers.

\section{Pathogenic bacteria-}

Microorganism that colonize in the mouth there are three,porphysomonasgingivalis,tannerella forsythia \&actinobacillusactinomycetemcomitans which have been implicated as etiologic agent in periodontitis .The presence of periodontal pathogens, though are necessary to cause disease, is not sufficient .The odd ratio of developing periodontal disease in an individuals who harbours the putative periodontal .pathogens is not high enough to consider them a risk factor ${ }^{(8)}$. The presence of A.actinomycetemcomitans confers no additional risk of developing localised aggressive periodontitis in adults despite the fact its presence is necessary for the disease to develop ${ }^{(9)}$. It is seen that provotellaintermedia ,p.gingivakis\&fusobacteriumnucleatum may be risk indicators for periodontal disease in a diverse population ,though they are not risk factor ${ }^{(10)}$

\section{Diabetes mellitus -}

Evidence shows that type $1 \& 2$ diabetes is more associated with periodontitis. In these patients, host response may be impaired, wound healing is delayed \&collagen lytic activity may be enhanced. Diabetes is a modified factor that though it cannot be cured, it can be controlled. Both positive \&negative conclusion has been seen in its relationship with periodontitis. In general no difference in impact has been determined between type $1 \& 2$ diabetes. Diabetic parameter examined includes glycaemiccontrol, duration of disease, presence of other diabetic associated complication \& population studied. Periodontal parameter examined have included gingivitis, clinical attachment loss, and alveolar bone loss. ${ }^{(11) .}$ A review of the literature by kinane found considerable evidence to suggest that diabetes and periodontitis have direct relationship (12) .Cross sectional studies on puma Indians, a group displaying the highest prevalence of type 2 diabetes in the world, show an odds ratio of 2.8 to 3.4 for developing periodontal disease in type 2 diabetic compared to non-diabetics ${ }^{(13)}$. Similarly, longitudinal studies have also shown increased risk of ongoing periodontal destruction in diabetic as compared to non-diabetics with an odd ratio of 4.2. Studies have been done which shows that poorly controlled diabetics respond less successfully to periodontal therapy relative to well controlled and non-diabetics ${ }^{(14)}$. Inflammation is a central feature of pathogenesis of diabetes and periodontitis. Periodontitis being an inflammatory disease is characterised by elevated levels of inflammatory mediators like TNF alfa, IL 6 and IL1beta, MMP- 8 , MMP-9 and cytokines. These locally produced pro- inflammatory mediators move into the systemic circulation and alters the glucose and lipid metabolism and inducing insulin resistance thus aggevating the condition. Evidence has consistently indicated that diabetes is a risk factor for increased severity of gingivitis and periodontitis. Conversely, periodontitis may be a risk factor for worsening glycaemic control among patients with diabetes. The first clear evidence to support this hypothesis came from investigations of individuals in the Gilia River Indian Community. Severe periodontitis was associated with an increased risk of poor glycaemiccontrol $(\mathrm{HbA} 1 \mathrm{c}>9.0 \%)$ at follow-up (minimum 2 years) suggesting that severe periodontitis was a risk factor for compromised diabetes management. Although there are several risk factors for periodontitis, diabetes mellitus has been until now, the only systemic disease connected through biochemical mechanism with periodontal disease (Gheric et al 2008). Another fact related to the relationship between periodontitis and diabetes, that may demonstrate it, is that diabeticsare moresusceptible to gingivitis and periodontitis than non- diabetic persons. Several authors have shown that metabolic disturbances in periodontitis decrease resistance to infections in diabetic patients, along with comes the initiation, development and progression of periodontal diseases.

\section{Risk determinants-}

Aging-

Ageing is associated with an increased incidence of periodontal disease ${ }^{(15)}$ However it has been suggested that the increased level of periodontal destruction observed with aging is the result of cumulative destruction rather than a result of increased rates of destruction. Thus aging is not a risk factor per se ${ }^{(16)}$ 
Gingivitis, in varying degrees, is nearly a universal finding in children and adolescent. Research has also observed some of the organism seen in periodontal disease in young children without signs of gum problems. One survey reported that $3.6 \%$ of adults between the ages of 18-34 year had had periodontal disease. As people age , the risk for periodontal disease increases. ${ }^{(27)}$

\section{Gender-}

Gender is known to for a modifier of the initiation and outcome of many conditions. Establishing whether sex difference exist in the development and progression of periodontitis is the important for both ,understanding pathogenesis and developing models of risk assessment for treatment planning taking in consideration effect of various systemic condition related to gender which can lead to periodontitis ${ }^{(26)}$.Gender plays a role in periodontal disease. There is evidence to support the higher prevalence of destructive periodontal disease in men than women. Men have poorer oral health than women, as evidenced by higher levels of plaque and calculus. The important factor to be considered is that women still have varied periodontal disease due to hormonal fluctuation in various decades of life.

\section{Genetic factor-}

Studies suggest that susceptibility to the early onset forms of disease, particularlyprepubertal and juvenile periodontitis, is , at at least in part, influenced by host genotype. In recent years ,genetic markers have become available to determine various genotype of patient regarding their susceptibility to periodontal disease .Research on the interleukin-1(IL-1) polymorphism has shown that IL-1 genotype positive patient shows more advanced periodontitis lesion that with IL-1 genotype negative patient of the same age group and there is a increased tooth loss in the IL-1 genotype positive subjects. In a retrospective analysis of over 300 well maintained periodontal patient, the IL-1genotype yield higher BOP\% during 1year recall period than the IL1 genotype negative control patient . This supports the theory that specific environmental factors can be strong risk factors and they overwhelm any genetically determined susceptibility or resistance to disease. ${ }^{(17)}$

\section{Stress-}

Stress has impact on the normal functioning of the immune system. Specific periodontal pathogens can utilize stress hormones to stimulate growth and expression of virulence factors providing another potential mechanism linking stress levels with periodontitis. Stress and related body distress are important risk indicator for periodontal disease .A recent study shows that people under physical or psychological stress are prone to elevated biofilm plaque levels and increased gingivitis and high levels of financial stress and poor coping abilities increases the likelihood of developing periodontal disease two fold. Systemic disease is associated with periodontal disease such as diabetes, cardiovasculardisease, preterm delivery and osteoporosis which may share psychological stress as a common risk factor .However, a direct association between periodontal disease and stress remains unproven.

The mechanism to elucidate how stress may affect periodontal disease is not known yet; however there are 2 pathways which may explain the role that stress is influencing on the onset and progression of periodontal disease. These include the biologic model and behaviouralmodel ${ }^{(18)}$. The biologic model proposes that periodontal disease may be biologically moderated through the hypothalamic-pituitary-adrenal(HPA) axis to promote the release of corticotropic releasing hormone from the hypothalamus and glucocorticosteroid, from the adrenal cortex. Behavioural model suggest that psychosocial stress may precipitate behavioural changes which affect at risk health behaviours. The possibility also exist that stress leads to overeating. Especiallyhigh fat diets, which can leads to immunosuppression through increased cortisol production. ${ }^{(19)}$

\section{Socioeconomic status-}

Socioeconomic status was represented by monthly family income. These groups were constituted and subjects were categorized into either lower class $(<1500-5000)$, middle class $(5000-15000) \&$ upper class $(15000 \&$ above). Presently very few studies shows that the effect of generaleducation, life style and socioeconomic position on the prevalence of periodontal disease. Hence in the present study an attempt was made to investigate the effect of lifestyle education, and socioeconomic position on the periodontal health status of a randomly selected adult population around Nellore India. Socioeconomic status was assessed by using a questionnaire followed by clinical recording of inflammation ,bleeding on probing ,periodontal pocket, and clinical attachment loss .Based on these findings, the patient were categorized into chronic generalised gingivitis(CGG),and chronic generalised periodontitis(CGP). ${ }^{(20)}$

\section{Risk indicator-}




\section{HIV/AIDS-}

The immunosuppression in HIV patients makes them highly susceptible to microbial infections. A multitude of oral lesion, including unique forms of periodontal disease , have been discovered in individuals infected with the immunodeficiency of virus(HIV).Periodontal disease appears to be less than previously thought ,many researchers agree that an important factor influencing the prevalence of unique periodontal disease in the HIV population is the degree of immunodeficiency. The pathogenesis of HIV associated periodontal disease is still unclear, but may be due to the result of micro biota and /or alteration in the host.HIV gingivitis, now called linear gingival erythema and HIV periodontitis, now called necrotizing ulcerative periodontitis, have microbiology profiles similar to conventional adult periodontitis, although these lesion are quite different clinically. ${ }^{(28)}$

\section{Osteoporosis-}

There is an association between osteoporosis and alveolar bone loss around a tooth ${ }^{(21)}$. Women with osteoporosis have reduction in bone mineral content, which increases the risk for fracture .Since the loss of alveolar bone around the tooth is a result of periodontal disease, osteoporosis is suspected to be related to periodontal disease ${ }^{(29)}$. According to WHO osteoporosis it is considered to be present when the bone mineral density (BMD)is 2.5 standard deviations below the young normal .Women are more susceptible to osteoporosis than men however ,osteoporosis in men,particularly at an older age is an important health problem in the elderly.Several cross sectional studies have shown that alveolar bone density is altered in osteoporotic individuals. Thereare fewer studies which demonstrate a relationship with clinical attachment levels. ${ }^{(22)}$

\section{Bleeding on probing-}

\section{Risk predictor-}

Bleeding on probing can be effective in the diagnosis and monitoring of active periodontal disease, ${ }^{(23)}$ but when used as a stand-alone test it can be accurate. A proper diagnosis should include a combination of bleeding on probing, probing depth and clinical attachmentloss ${ }^{(30)}$. Bleeding on probing is useful for predicting the progression of periodontitis, and pockets that bleed on two or three consecutive appointment are likely to become active .Gingival bleeding can also be symptomatic of systemic issues, and unless these are treated correctly, periodontal therapy may not be sufficient to restore oral health. Smokers may exhibit less bleeding on probing because nicotine can supress bleeding ,causing vasoconstriction in peripheral blood vessels that can lead to a compromised immune response. In 1986, a study introduced experimental gingivitis to a group of dental students ,half of whom were smokers. ${ }^{(24)}$

\section{Previous history of periodontal disease-}

Patient with history of previous gum disease are found to be more prone to periodontal disease. Frequent dental check-up from oral health professionals is advised for preventing aggravation of risk factor causing the disease. History of periodontal disease should be elicited before commencing the treatment protocol for periodontitis.

\section{Conclusion-}

The prevention and treatment of periodontitis is therefore based on accurate diagnosis and risk management. Smoking and type I \& II diabetes are well -established risk factor for periodontal disease, whereas the etiologic microorganism P.gingivalis ,T.forsythia and A. actnomycetemcomitans are risk indicators. Genetic polymorphism, especially with regard to IL1 but also tumour necrosis factor TNF , human leukocyte antigen and others have also been investigated, although strictly speaking they cannot be generally considered risk markers or risk indicators ${ }^{(25)}$. Psychological stress is considered a risk indicator for periodontal disease. Evaluation of these risk factors should be considered and the patient should be educated concerning their risk and an appropriate prognosis and treatment plan should be implemented.

\section{Reference-}

[1]. TimmermanMF,Van der Weijden.Risk factors for periodontitis .Int J Dent Hyg.2006;4:2-7[PubMed]

[2]. Albandar et al.,2000 J IntAcad periodontal .2005 January ;7(1):3-7. Risk Factor for periodontitis

[3]. BergstromJ,EliassonS.Jack Dock Exposure to tobacco smoking and periodontal health .J Clin Periodontol .2000;27:61-8[PubMed]

[4]. Bergstrom and bostrom ,2001;Muller et al .,2001 J IntAcad periodontal .2005 January ;7(1):3-7. Risk Factor for periodontitis

[5]. Bergstrom ,1999 J IntAcad periodontal .2005 January ;7(1):3-7.Risk Factor for periodontitis

[6]. bostram et al.,1998;Ah,et al.,1994 J IntAcad periodontal .2005 January ;7(1):3-7.Risk factor for periodontitis

[7]. Ryder et al.,1998a;Ryder et al.,1998b J IntAcad periodontal .2005 January;7(1):3-7.Risk factor for periodontitis

[8]. Ezzo and Cutler ,2003 J IntAcad periodontal.2005January;7(1):3-7.Risk Factor for periodontitis

[9]. Buchmann ,et al.,2000 J IntAcad periodontal.2005 January;7(1):3-7.Risk Factor for periodontitis

[10]. Alpagot et al.,1996 J IntAcad periodontal .2005 January;7(1):3-7.Risk factor for periodontitis

[11]. Tomar and Asma,2000 J IntAcad periodontal 2005 january ;7(1):3-7.Risk Factor for periodontitis 
[12]. Kinane and chestnut, 1997 J IntAcad periodontal 2005 January;7(1):3-7.Risk Factor for periodontitis

[13]. Emrich et al.,1991 J IntAcad periodontal 2005 January;7(1):3-7.Risk factor for periodontitis

[14]. Westfelt et al.,1996;tervonan and karjalainen,1997 J IntAcad periodontal 2005 January:7(1):3-7.Risk factor for periodontitis

[15]. Grossi et al.,1994; Grossi et al .,1995 J IntAcad periodontal 2005 January;7(1):3-7.Risk factor for periodontitis

[16]. Genco,1996 J IntAcad periodontal 2005 January;7(1)3-7.Risk factor for periodontitis

[17]. LangNP,TonettiMS.Periodontal Risk assessment (PRA) for patients in supportive periodontal therapy (SPT) oral health Prev Dent.2003;1:7-16.[PubMed]

[18]. Genco RJ, Ho AW kopmenJ,etal.models to evaluate the role of stress in PO. Ann periodontal 1998;3:288-302

[19]. RoseLF,Mealey,BL ,GencoRJ,CohenDW.periodontics :medicine, surgery \& implants. 2004,Elsevier mosby,pg. 818

[20]. Contemp Clin Dent.2010 jan - mar ;1(1):23-26 effect of life style, education and socioeconomic status on periodontal health

[21]. JeffcoatMK.Osteoporosis :A Possible modifying factor in oral bone loss .Ann Periodontal 3:312-321,1998.

[22]. Thomas E Van Dyke, SaileshDave.RJJntAcad periodontal .2005 january ; 7(1): 3-7 Risk factor for periodontitis

[23]. ClaffeyN,Engelberg J. Clinical Indicator of probing attachment loss following initial periodontal treatment in advanced periodontitis patient .J Clin periodontal $1995 ; 22: 690-696$

[24]. BergstromJ,preberH.The influence of cigarette smoking on the development of experimental gingivitis .J periodontal Res.1986;21:668-676.

[25]. Thomas E et el .J IntAcad Periodontal .2005 january ;7(1):3-7 Risk factors for periodontitis

[26]. NazishAlam ,Priyam Mishra ,SC Chandrasekaran .Gender Basis of Periodontal Disease:Indian Journal Of Basic and Applied Medical research ;March $2012: 128-135$

[27]. Periodontitis Risk Factor -health Guide - The Newyork Times

[28]. Murray PA. HIV disease as a risk factor for periodontal disease ;Compendium .1994 Aug;15(8):1052,1054-63 ;quiz1064

[29]. MealeyBL.Periodontal implication :Medically compromised patient .Ann Periodontal 1:256-321.1996

[30]. LindheJ,Haffajee AD, SocranskySS.progression of periodontal disease in adult subjects in the absence of periodontal therapy .J Clin periodontal 1983;10433-442 